Supporting Information (SI) concerning the manuscript:

\title{
Spin Control in Ladder-Like Hexanuclear Copper(II) Complexes with Metallacyclophane Cores
}

Emilio Pardo, Kevin Bernot, Miguel Julve, Francesc Lloret, ${ }^{*}$ Joan

Cano, Rafael Ruiz-García, Fernando S. Delgado, Catalina RuizPérez, Xavier Ottenwaelder, and Yves Journaux*

Departament de Química Inorgànica, Instituto de Ciencia Molecular, and Departament de Química Orgànica, Facultat de Química, Universitat de València, 46100 Burjassot, València, Spain, Laboratorio de Rayos X y Materiales Moleculares, Departamento de Física Fundamental II, Universidad de La Laguna, 38204 La Laguna, Tenerife, Spain, and Laboratoire de Chimie Inorganique, Université Paris-Sud, 91405 Orsay, France 


\section{Experimental Section}

General. All chemicals were of reagent grade quality, and they were purchased from commercial sources and used as received. IR spectra were recorded on a Perkin-Elmer 882 spectrophotometer as $\mathrm{KBr}$ pellets. UV/Vis solution spectra were recorded on a PerkinElmer Lambda 2 spectrophotometer. Elemental analyses $(\mathrm{C}, \mathrm{H}, \mathrm{N})$ were performed by the Microanalytical Service of the Universidad Autónoma de Madrid (Spain). The starting materials $\mathrm{Na}_{4}\left[\mathrm{Cu}_{2}(\mathrm{mpba})\right] \cdot 8 \mathrm{H}_{2} \mathrm{O}$ and $\mathrm{Li}_{4}\left[\mathrm{Cu}_{2}(\mathrm{ppba})\right] \cdot 10 \mathrm{H}_{2} \mathrm{O}$ were obtained by literature methods.

$\left\{\left[\mathrm{Cu}_{2}(\text { mpba })_{2}\right][\mathrm{Cu}(\text { tmen })]_{4}\right\}\left(\mathrm{ClO}_{4}\right)_{4} \cdot \mathbf{6 H}_{2} \mathbf{O}$ (3): An aqueous solution $(10 \mathrm{~mL})$ of $\mathrm{Na}_{4}\left[\mathrm{Cu}_{2}(\mathrm{mpba})\right] \cdot 8 \mathrm{H}_{2} \mathrm{O}(0.43 \mathrm{~g}, 0.5 \mathrm{mmol})$ was added to a solution of copper(II) perchlorate hexahydrate $(0.74 \mathrm{~g}, 2.0 \mathrm{mmol})$ and $N, N, N^{\prime}, N^{\prime}$-tetramethylethylenediamine $(0.30 \mathrm{~mL}, 2.0$ $\mathrm{mmol})$ in water $(20 \mathrm{~mL})$. Slow evaporation of the deep blue filtered solution at room temperature gives a polycrystalline dark green powder after a few days, which was filtered off and air-dried. Anal.: calcd for $\mathrm{C}_{44} \mathrm{H}_{84} \mathrm{Cl}_{4} \mathrm{Cu}_{6} \mathrm{~N}_{12} \mathrm{O}_{34}$ (1847): C, 28.59; H, 4.55; N, 9.10. Found: C, 28.17; H, 4.44; N, 9.23; IR (KBr) 3500, 3465, 3392 (OH), 1624, $1616(\mathrm{CO}) \mathrm{cm}^{-}$ 1; UV/Vis $\left(\mathrm{H}_{2} \mathrm{O}\right) \lambda_{\text {max }}\left(\varepsilon / \mathrm{M}^{-1} \mathrm{~cm}^{-1}\right) 270$ (30600), 390 (sh) (2800), 655 (450) nm. Up to now, all our attempts to grow single crystals by recrystallization from water were unsuccessful.

$\left\{\left[\mathrm{Cu}_{2}(\mathbf{p p b a})_{2}\right][\mathrm{Cu}(\mathbf{p m d i e n})]_{4}\right\}\left(\mathrm{ClO}_{4}\right)_{4}(\mathbf{4}):$ An aqueous solution $(10 \mathrm{~mL})$ of $\mathrm{Li}_{4}\left[\mathrm{Cu}_{2}(\mathrm{ppba})\right] \cdot 10 \mathrm{H}_{2} \mathrm{O}(0.42 \mathrm{~g}, 0.5 \mathrm{mmol})$ was added to a solution of copper(II) perchlorate hexahydrate $(0.74 \mathrm{~g}, 2.0 \mathrm{mmol})$ and $N, N, N^{\prime}, N$ ”, $N$ ”-pentamethyldiethylenetriamine $(0.42$ $\mathrm{mL}, 2.0 \mathrm{mmol})$ in water $(20 \mathrm{~mL})$. X-ray quality dark green prisms of $\mathbf{4}$ were obtained after several days of slow evaporation at room temperature of the deep blue filtered solution. Anal.: calcd for $\mathrm{C}_{56} \mathrm{H}_{100} \mathrm{Cl}_{4} \mathrm{Cu}_{6} \mathrm{~N}_{16} \mathrm{O}_{28}$ (1969): C, 34.16; H, 5.08; N, 11.39. Found: C, 34.08; $\mathrm{H}, 5.14$; N, 11.33; IR (KBr) $1622(\mathrm{CO}) \mathrm{cm}^{-1}$; UV/Vis $\left(\mathrm{H}_{2} \mathrm{O}\right) \lambda_{\max }\left(\varepsilon / \mathrm{M}^{-1} \mathrm{~cm}^{-1}\right) 290$ (38500), 415 (sh) (2850), 660 (1025) nm. 
Magnetic measurements. Variable-temperature $(1.8-300 \mathrm{~K})$ magnetic measurements under an applied magnetic field of $1 \mathrm{~T}$ were carried out on powdered samples of $\mathbf{3}$ and $\mathbf{4}$ with a SQUID magnetometer. The magnetic susceptibility data were corrected for diamagnetism of the constituent atoms (Pascal's constants) and also for temperatureindependent paramagnetism $\left(60 \times 10^{-6} \mathrm{~cm}^{3} \mathrm{~mol}^{-1}\right.$ per copper atom). 